\title{
Mechanistic and biological significance of DNA methyltransferase 1 upregulated by growth factors in human hepatocellular carcinoma
}

\author{
QIN-LIANG FANG $^{1 *}$, YI-RUI YIN $^{1 *}$, CHENG-RONG XIE $^{1 *}$, SHENG ZHANG $^{1}$, \\ WEN-XIU ZHAO ${ }^{1}$, CHAO PAN $^{2}$, XIAO-MIN WANG $^{1}$ and ZHEN-YU YIN ${ }^{1}$ \\ ${ }^{1}$ Department of Hepatobiliary Surgery, Zhongshan Hospital of Xiamen University, \\ Fujian Provincial Key Laboratory of Chronic Liver Disease and Hepatocellular Carcinoma, Xiamen, Fujian 361004; \\ ${ }^{2}$ Department of Pathology, Zhongshan Hospital of Xiamen University, Xiamen, Fujian 361004, P.R. China
}

Received September 15, 2014; Accepted October 30, 2014

DOI: $10.3892 /$ ijo.2014.2776

\begin{abstract}
Dysregulation of growth factor signaling plays a pivotal role in controlling the malignancy phenotype and progression of hepatocellular carcinoma (HCC). However, the precise oncogenic mechanisms underlying transcription regulation of certain tumor suppressor genes (TSGs) by growth factors are poorly understood. In the present study, we report a novel insulin-like growth factor 1 (IGF1) pathway that mediates de novo DNA methylation and TSG (such as DLCl and CHD5) silencing by upregulation of the DNA methyltransferase 1 (DNMT1) via an AKT/ $\beta$-transducin repeat-containing protein $(\beta \mathrm{TrCP})$-mediated ubiquitin-proteasome pathway in HCC. Analysis of DNA methylation in $\mathrm{CpG}$ islands of target genes revealed high co-localization of DNMT1 and DNMT3B on the promoters of TSGs associated with enhanced $\mathrm{CpG}$ hypermethylation. Our results point to a novel epigenetic mechanism for growth factor-mediated repression of TSG transcription that involves DNA methylation.
\end{abstract}

\section{Introduction}

Hepatocellular carcinoma (HCC), the most common primary liver tumor, is the fifth most common human malignancy and the second leading cause of cancer death $(695,900$ deaths annually) in men worldwide (1). Owing to difficulty in early diagnosis and a lack of effective chemotherapy and radiotherapy, the 5-year overall survival rate remains poor for patients with advanced HCC (2-4). Changes in growth factors, receptors and their downstream signaling pathway components

Correspondence to: Dr Zhen-Yu Yin, Department of Hepatobiliary Surgery, Zhongshan Hospital of Xiamen University, 209 South Hubin Road, Xiamen, Fujian 361004, P.R. China

E-mail: yinzy@xmu.edu.cn

*Contributed equally

Key words: DNA methyltransferase 1, DNA methylation, hepatocellular carcinoma, insulin-like growth factor 1 influence HCC development and malignancy. The signaling pathways involved include IGF-IGF1R, hepatocyte growth factor (HGF/MET), EGF-EGFR and TGF $\beta$-T $\beta$ R signaling (5). Ligands bind to their corresponding receptors, which are usually overexpressed and/or dysregulated in HCC, and mainly activate the PI3K-PKB (AKT) and MAPK pathways, which affect tumor cell phenotypes (5-8). IGF secreted by liver cells acts in an autocrine and/or paracrine manner by binding to IGF1R (9). This in turn initiates downstream cellular effectors including insulin-receptor substrates and Src homology domain-containing kinases, ultimately contributing to proliferation, anti-apoptosis, invasiveness and metastasis (5,10-13). Growth factors that are dysregulated in tumorigenesis and cancer progression represent rational targets for HCC therapies. Nevertheless, the precise oncogenic mechanisms by which growth factors affect the transcriptional regulation of certain tumor suppressor genes (TSGs) are unclear.

Epigenetic mechanisms play an important role in driving tumorigenesis. DNA methylation catalyzed by DNA methyltransferases (DNMTs), causes changes in chromatin structure, DNA conformation and stability and patterns of DNA-protein interaction. DNA hypermethylation leads to transcriptional silencing of a considerable number of TSGs; thus, hypermethylation has been proposed as the second hit in a model of TSG inactivation (14). Overexpression of DNMTs has been observed in various types of cancers (15-20), and is significantly correlated with poor histological differentiation and prognosis. These findings suggest that aberrant DNA hypermethylation drives hepatocarcinogenesis. It is recognized that many risk factors for HCC may upregulate DNMTs expression, such as HBV-encoded protein X (21). The tobacco-specific carcinogen NNK (nicotine-derived nitrosamine ketone) induces DNMT1 protein upregulation and binding to the promoters of various TSGs, which ultimately leads to tumorigenesis and poor prognosis in lung cancer (22). These findings shed light on the relevance of the expression of DNMTs in carcinogenesis. However, there is little evidence on the delicate mechanism of DNMTs overexpression in HCC. The findings presented here highlight the mechanistic significance of aberrant DNMT1 expression induced by IGF1 in HCC. 


\section{Materials and methods}

Cell culture. HCC cell lines (HepG2, BEL-7402 and SMMC-7721) were obtained from the Type Culture Collection of the Chinese Academy of Sciences (Shanghai, China). The HL-7702 immortalized normal human liver cell line was obtained from the China Center for Type Culture Collection (Wuhan, China). Cell lines were cultured in Dulbecco's modified Eagle's medium (DMEM; Gibco) or RPMI-1640 medium (Gibco) supplemented with 10\% fetal bovine serum (FBS; HyClone) and $1 \%$ penicillin-streptomycin.

Quantitative RT-PCR ( $q R T$-PCR). qRT-PCR was performed as previously described (23) using an ABI PRISM 7300 detection system. qRT-PCR reactions were performed using SYBR-Green (Roche) technology and were repeated at least three times.

Flow cytometry and cell cycle synchronization assays. HepG2 cells were seeded in DMEM (0.1\% FBS) medium at a density of $5 \times 10^{5}$ cells $/ 35-\mathrm{mm}$ dish. The cells were treated with DMSO or $15 \mu \mathrm{M}$ 5-aza-2-deoxycytidine (5'-Aza; Sigma-Aldrich) in time-course experiments. Cell suspensions in PBS were prepared for analysis using a FlowCellect Bivariate Cell Cycle kit (Millipore) according to the manufacturer's protocol. At various time-points after release, cells were pulsed with $10 \mu \mathrm{M}$ BrdU for $2 \mathrm{~h}$ immediately before harvesting and fixation. Cell pellets were processed for double staining with an anti-BrdU antibody (Millipore) and propidium iodide (PI, $10 \mu \mathrm{g} / \mathrm{ml}$; Millipore), followed by analysis on a flow cytometer (Partec CyFlow Space). Gating was performed to focus on G1, S and $\mathrm{G} 2 / \mathrm{M}$ populations.

In vivo tumorigenicity assays and 5'-Aza treatment. Female athymic nude mice (Vital River Animal Center, Beijing, China) were housed in a temperature-controlled sterile room in which humidity and light were carefully controlled and monitored. Animal welfare and experimental procedures were in strict accordance with the regulations approved by the Institutional Animal Care Committee of the Medical College at Xiamen University. HepG2 cells $\left(5 \times 10^{6}\right)$ were injected subcutaneously into the flank of 6-week-old nude mice. When the tumor size reached $0.5 \mathrm{~cm}^{3}$, the mice were randomly divided into two groups and subjected to the following treatments: i) i.p. injection of PBS as a control; ii) $1 \mathrm{mg} / \mathrm{kg}$ 5'-Aza was given by i.p. injection every 2 days over a treatment time of 14 days (seven injections). Tumor volume was calculated as volume $=$ width $^{2}$ $\mathrm{x}$ length $\mathrm{x} 0.52 \mathrm{~mm}^{3}$.

ChIP-on-chip and target gene verification. The protocol provided by NimbleGen was followed. In brief, HepG2 cells were grown, cross-linked with formaldehyde and sheared by sonication. Antibodies (DNMT1, DNMT3B and IgG; Santa Cruz Biotechnology) were used for immunoprecipitation. A HG18 RefSeq Promoter microarray (NimbleGen) was used for hybridization and analysis according to the manufacturer's instructions. Data extraction was performed using NimbleScan version 2.5 and significant hybridization peaks (FDR $<0.05$ ) were identified. For target gene verification we used real-time PCR and a chromatin immunoprecipitation (ChIP) assay.
ChIP and DNA methylation assay. The two experiments were performed as previously described with slight modification $(24,25)$.

Statistical analysis. SPSS 15.0 software (SPSS, Inc., Chicago, IL, USA) was used for all statistical analyses. Data from cell growth and mouse tumorigenesis experiments were analyzed by one-way ANOVA. To analyze differences in DNMT1 protein levels among various cell treatments, a two-tailed Student's t-test was used. Survival curves were calculated by the Kaplan-Meier method, and comparison was performed using a log-rank test. Results for parametric variables are expressed as mean \pm SE. In all cases, $\mathrm{P}<0.05$ was considered to indicate a statistically significant result.

\section{Results}

Identification of target genes of DNMT1 and DNMT3B. To screen for genomic DNA methylation profiles and potential target genes in HCC, we carried out ChIP-on-chip coupled with $\mathrm{CpG}$ island microarray (MCAM) analysis in HepG2 cells. Extensive genome-wide DNA methylation of $\mathrm{CpG}$ islands of target genes was determined, with high co-localization of DNMT1 and DNMT3B; results for chromosome 1 are shown in Fig. 1A. We identified 2,177 genes with promoter hypermethylation in HepG2 cells, and 3,117 and 1,999 genes affected by DNMT1 and DNMT3B, respectively. Of these, 520 and 280 genes shared a methylation chip with DNMT1-ChIP or DNMT3B-ChIP, respectively. Among all three groups, 275 genes were screened out (Fig. 1B). Consistent with the above results, unsupervised hierarchical clustering analysis demonstrated that the top 32 genes with promoter hypermethylation in ChIP-on-chip results are closely related to tumor development and progression (Fig. 1C). However, we also found that DNA methylation of some gene sites does not rely on DNMT1 or DNMT3B (data no shown), implying the potential DNA methylation role of other DNMTs in HCC, such as DNMT3A; this will be the subject of further investigation.

Knockdown of DNMTs inhibit HCC malignant phenotype. We focused on the functional effect of DNMTs in controlling HCC malignant phenotype. MTT assay results show that transient inhibition of either DNMT1 or DNMT3B by siRNA was sufficient to reduce HepG2 cell proliferation (Fig. 2A and B). Strikingly, both siRNAs cooperatively suppressed HepG2 cell proliferation after 6 days and had a more pronounced effect than either siRNA alone (Fig. 2B). Time-course cell cycle analysis revealed that 5'-Aza inhibited HepG2 cell cycle progress from $\mathrm{G} 1$ phase to $\mathrm{S}$ phase (Fig. 2C). Moreover, 5'-Aza treatment inhibited HepG2 cell migration (Fig. 2D; $\mathrm{P}=0.003$ ). To delineate the role of DNA methylation in HCC growth in vivo, we performed xenograft experiments in which HepG2 cells were injected subcutaneously into immunodeficient nude mice. The tumor growth rate significantly decreased after treatment with 5'-Aza compared to control animals. The average tumor volume was $1.96 \mathrm{~cm}^{3}$ in the control group on day 28 compared to only $1.23 \mathrm{~cm}^{3}$ in the group treated with 5'-Aza (Fig. 2E; $\mathrm{P}=0.001$ ). These results argue that DNMTs cooperatively mediate DNA methylation and broadly participate in controlling the malignant phenotype of HCC cells. 


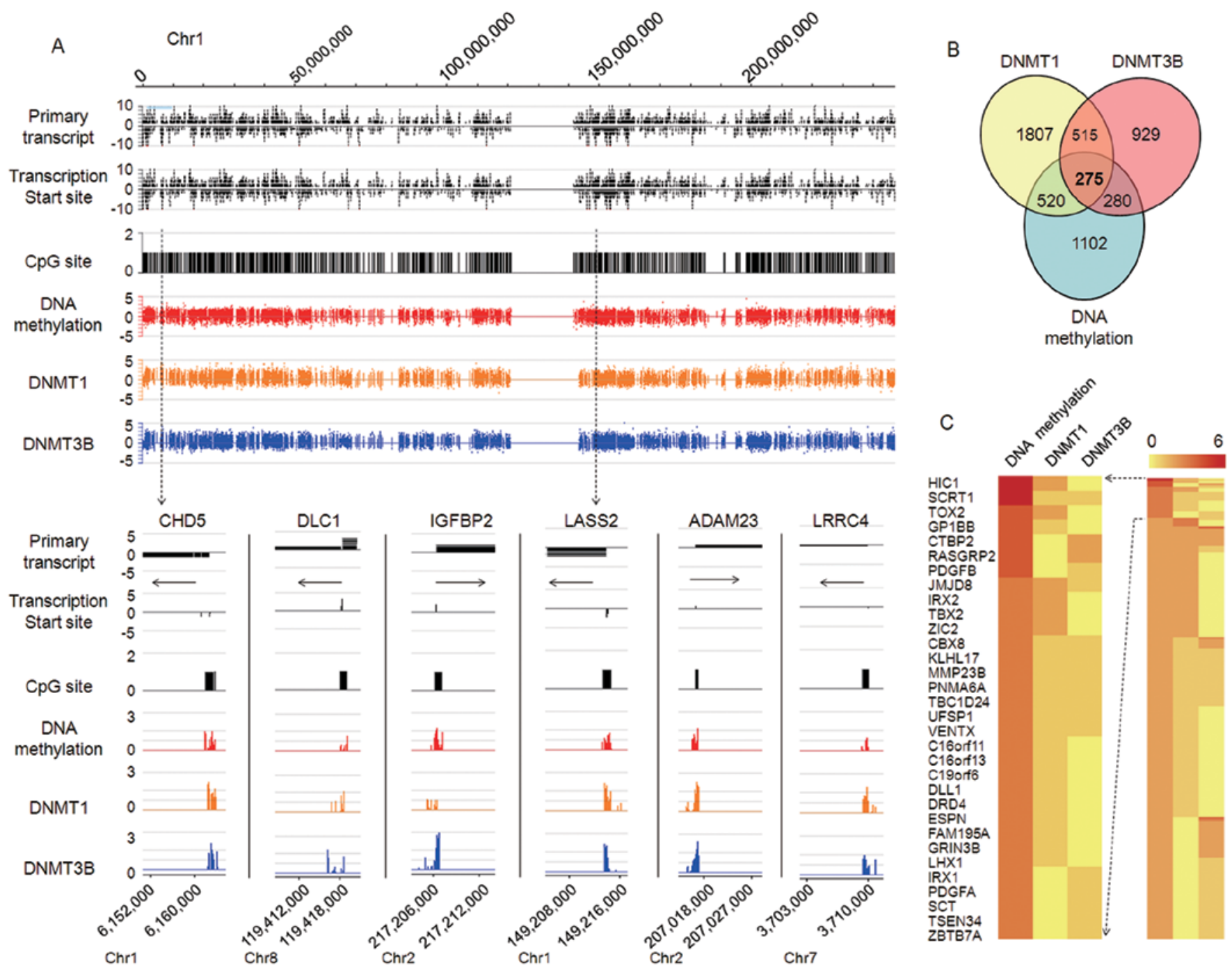

Figure 1. Genome-wide analysis of CpG island methylation and DNMTs occupancy in HepG2 cells. (A) ChIP-on-chip of DNMTs and methylation pattern on chromosome 1 (top) and expanded view of some positive signals for tumor suppressors from chromosome 1 and other chromosomes (bottom). (B) Venn diagram analysis of the methylated genes and DNMT1/DNMT3B target genes in the indicated categories. (C) Gene tree view of the MCAM analysis and ChIP-on-chip DNMT results. Hierarchical clustering was performed. Each row represents a single gene.

DNMT1 protein expression is upregulated by growth factors. Epigenetic regulation is sensitive to the environment, including extracellular environmental factors. We hypothesized that DNA methylation is influenced by growth factor pathways in HCC cells. To dissect the potential relationship between HCC-related growth factors and DNMT1 expression, we determined the effect of IGFs, HGF and EGF on DNMT1 expression in HCC cells. The results from western blotting clearly show that DNMT1 protein expression was upregulated by IGF1 stimulation in a dose-dependent manner in HepG2 cells (Fig. 3A). Time course experiments revealed that $50 \mathrm{ng} / \mathrm{ml}$ IGF1 increased DNMT1 expression in HepG2, BEL-7402 and HL-7702 cells (Fig. 3B). Furthermore, HGF and EGF increased DNMT1 protein expression (data no shown). These results point to a novel pathway of HCC-related growth factors that act by upregulating DNMT1, and suggest that DNMT1 has an important biological function in regulating HCC malignant phenotype.

We investigated the involvement of the IGF-IGF1R $\beta$ pathway in the regulation of DNMT1 expression. The
PI3K-AKT pathway is a key downstream signal of the IGF-IGF1R $\beta$ activation. To confirm whether this pathway is responsible for DNMT1 upregulation by IGF1, experiments to clarify which signaling pathway was blocked were performed. No obvious inhibition of DNMT1 expression was observed after treatment with $10 \mu \mathrm{M}$ of LY-294002 (Fig. 3C, lane 3). However, 10 and $50 \mu \mathrm{M}$ of LY-294002 significantly suppressed IGF1-induced DNMT1 expression, with downregulation of pAKT and pGSK3 (Fig. 3C, lanes 4 and 6). Notably, blocking of the PI3K-AKT pathway by LY-294002 led to a marked increase in IGF1-induced pIGF1R $\beta$ phosphorylation (not necessarily activation) in a dose-dependent manner (Fig. 3C, lanes 2, 4 and 6), indicating the pivotal role of the PI3K-AKT pathway in IGF-IGF1R $\beta$ activation signal transduction in the HepG2 cells. Furthermore, we found that PI3K-AKT phosphorylation was effectively stimulated by IGF2, HGF and EGF (data no shown). Taken together, the results indicate the transmission effects of the PI3K-AKT pathway on IGF-IGF1R $\beta$-induced DNMT1 protein expression. 
A

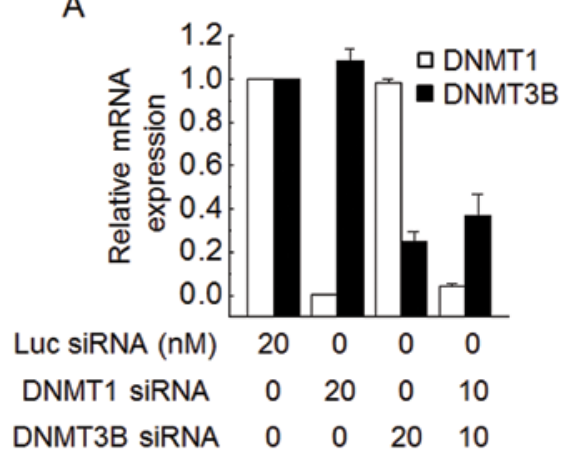

B

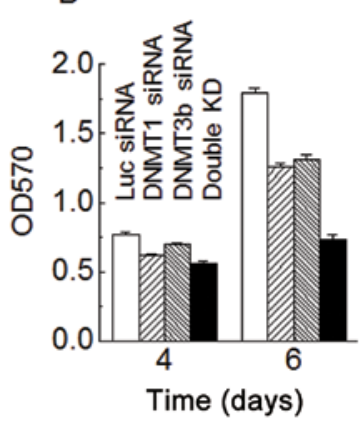

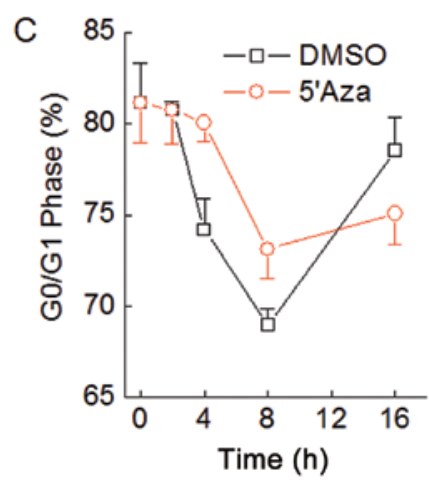

$\mathrm{D}$

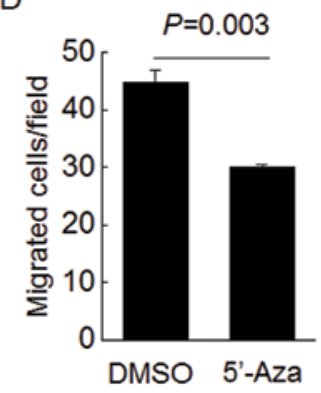

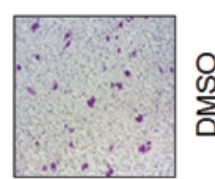

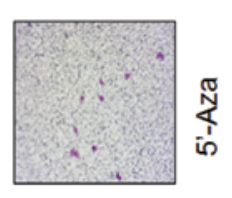

$\mathrm{E}$

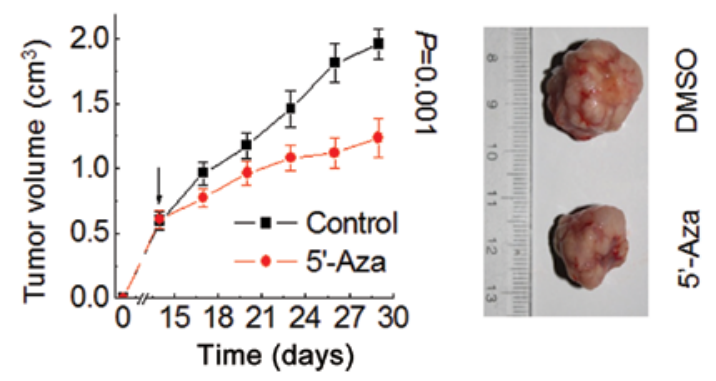

Figure 2. Knockdown of DNMTs inhibit HCC malignant phenotype. (A) Effects of siRNAs on the silencing of DNMT genes in HepG2 cells. Cells were transfected with DNMT1 siRNA and/or DNMT3B siRNA. Total mRNA was harvested and quantitative real-time PCR assays were performed for DNMT1 and DNMT3B. The relative levels of DNMT1 and DNMT3B mRNAs were normalized to $\beta$-actin. (B) After DNMT1 or/and DNMT3B siRNA transfection, survival of HepG2 cells harvested at the time-points indicated and assessed in an MTT assay. Data are mean \pm SE $(n=3)$. (C) Effect of DNMTs on cell cycle kinetics in HepG2 cells. Cell cycle distribution was determined using BrdU/PI bivariate analysis by flow cytometry. The percentage of 5'-Aza-treated and control HepG2 cells in the G0/G1 phase is shown as the mean \pm SE for three independent experiments. (D) Representative images of migration in 5'-Azatreated HepG2 and control cells in Transwell assays. ( $\mathrm{P}=0.003$; independent Student's t-test). (E) Tumor growth curves for 5'-Aza-treated HepG2 cells in vivo. The curves show tumor growth up to day 28 , when all mice were sacrificed. $\mathrm{P}=0.001$ compared to controls (PBS injection).

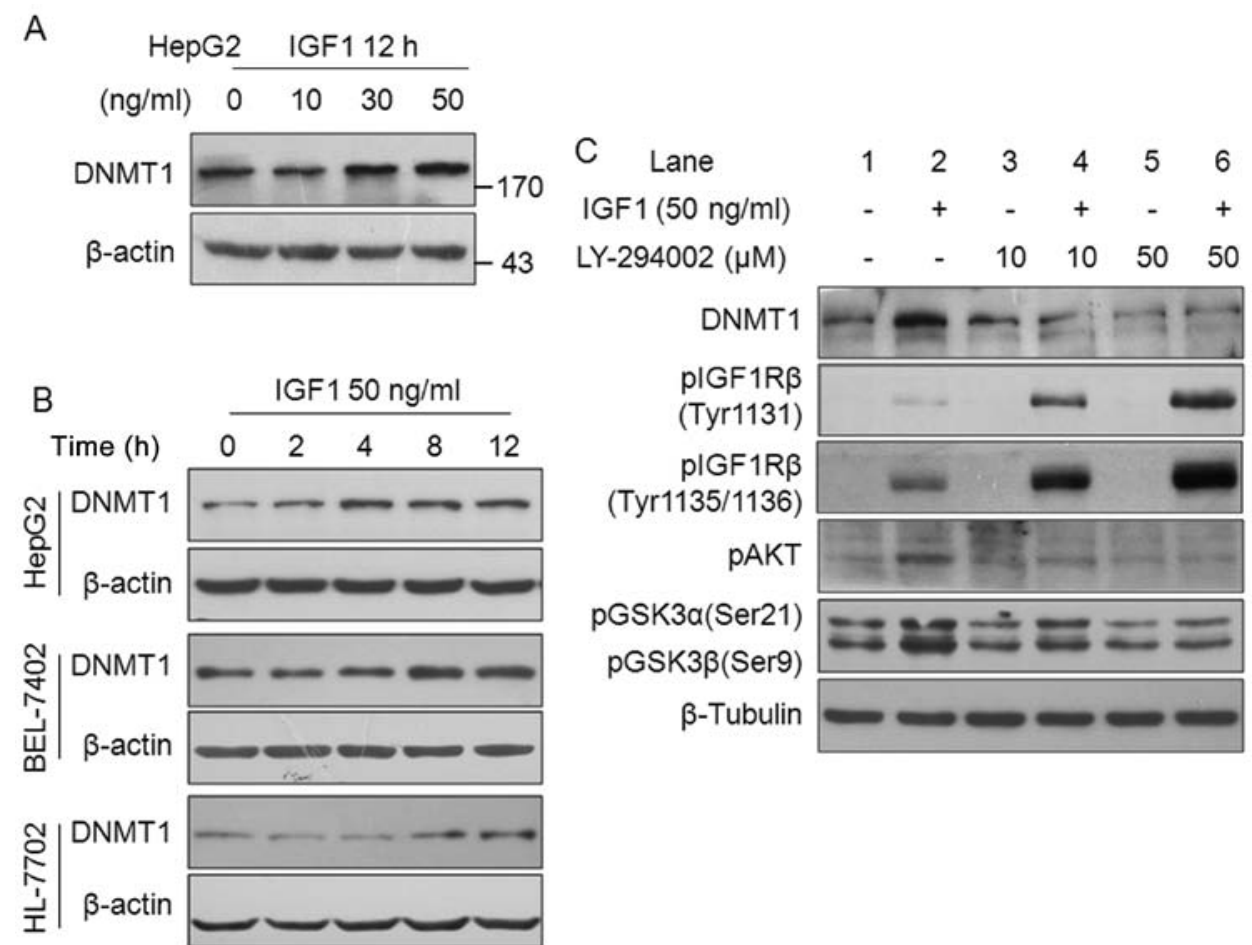

Figure 3. Growth factors upregulate DNMT1 protein levels. (A) Expression levels of DNMT1 in HepG2 treated with IGF1 were analyzed by western blotting as a function of dose. (B) Expression levels of DNMT1 in human liver cell lines treated with IGF1 were analyzed by western blotting as a function of time. (C) HepG2 cells were pretreated with or without the PI3K-AKT pathway inhibitor LY-294002 for $2 \mathrm{~h}$, followed by IGF1 treatment for $4 \mathrm{~h}$. Western blots show the protein levels of IGF1-induced DNMT1, pIGF1R $\beta$, pAKT and pGSK3 $\beta$. 


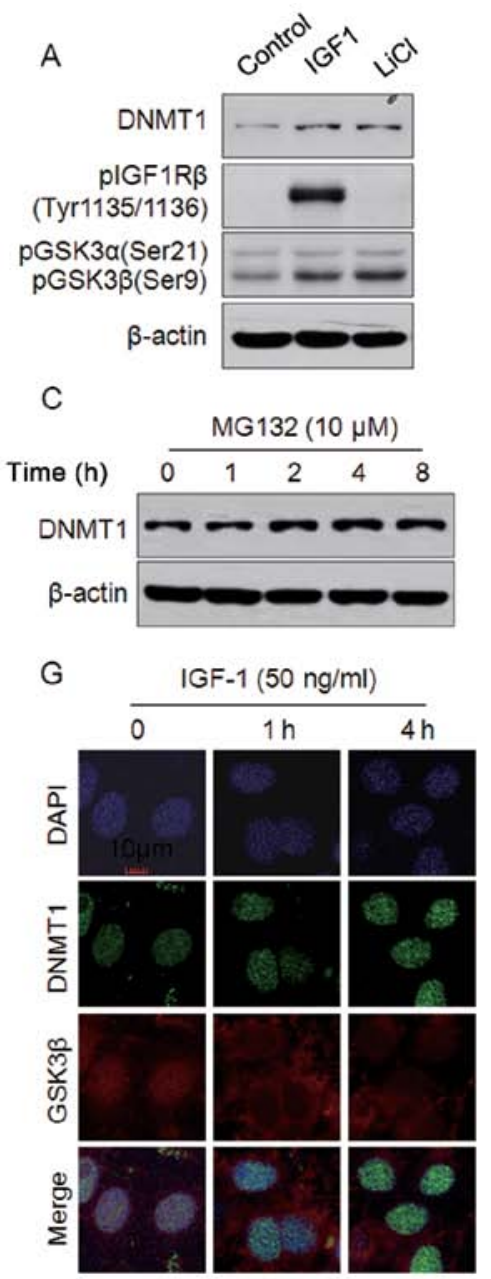

B

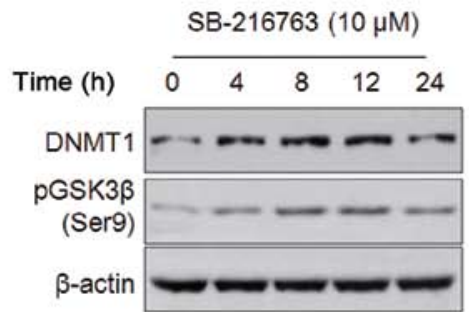

D

$\begin{array}{lccccccc}\text { IGF1 } & - & - & - & - & + & + & + \\ \text { CHX } & 0 & 12 & 24 & 36 & 12 & 24 & 36 \mathrm{~h}\end{array}$

DNMT1

$\beta$-actin

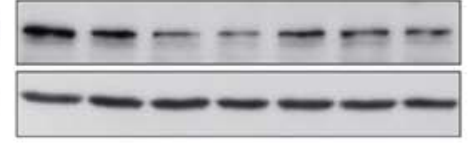

$\mathrm{H}$

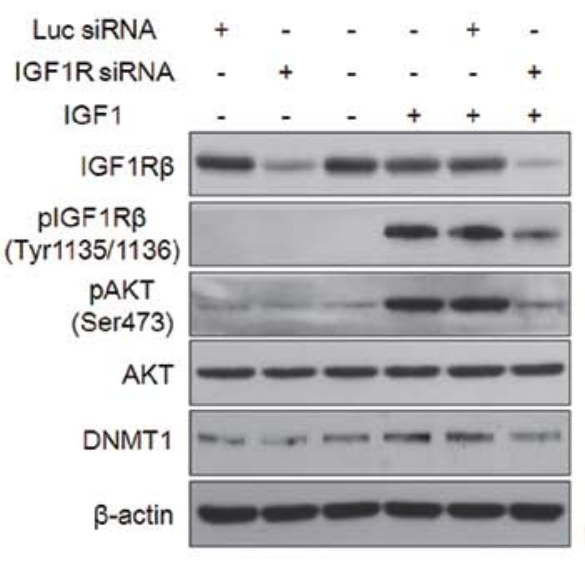

$E$
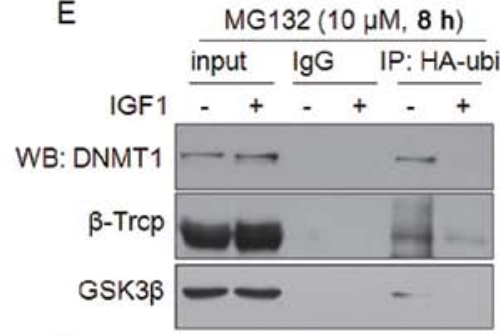

$\mathrm{F}$
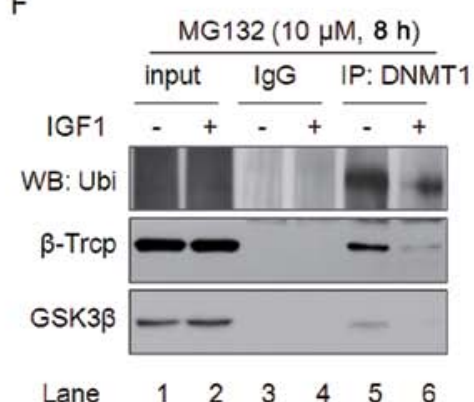

Figure 4. IGF1 inhibits DNMT1 protein degradation via ubiquitination chiefly through the PI3K-AKT-GSK3 $\beta$ pathway. (A and B) HepG2 cells were treated with the GSK3 $\beta$ inhibitors (A) $20 \mathrm{M} \mathrm{LiCl}$ for $4 \mathrm{~h}$ and (B) SB216763 for the time indicated and protein levels of DNMT1 and pGSK3 $\beta$ were detected by western blotting. (C) HepG2 cells were exposed to MG132 for 0-8 h and protein levels of DNMT1 were detected by western blotting. (D) Western blotting of DNMT1 protein levels in serum-starved HepG2 cells exposed to IGF1 with or without CHX pretreatment. (E and F) HepG2 cells were pretreated with MG132 after transfection with a HA-ubiquitin/DNMT1 construct, then treated with IGF1 for $4 \mathrm{~h}$. Cell lysates were immunoprecipitated with anti-HA or anti-DNMT1 and then western blotting was performed for DNMT1/ubiquitin, GSK3 $\beta$ and $\beta$ TrCP proteins. Normal IgG was used as a negative control. (G) GSK3 $\beta$ (red), DNMT1 (green) and 4,6-diamidino-2-phenylindole (DAPI) (blue) were detected by IF (magnification, x1600) in serum-starved HepG2 cells treated with IGF1. (H) Cell lysates from HepG2 cells treated with or without IGF1 $(50 \mathrm{ng} / \mathrm{ml})$ for $4 \mathrm{~h}$ were subjected to SDS-PAGE and analyzed for DNMT1 expression and activation of IGF1R and AKT. (I) Protein expression levels of DNMT1 and pAKT in HCC samples were detected by western blotting.

IGF1 inhibits ubiquitin-proteasome-mediated degradation of DNMT1 protein by GSK3 $\beta-\beta T$ TrCP complexes. Next, we focused on how the PI3K-AKT pathway affects IGF1-IGF1R $\beta$ axis-induced DNMT1 expression. IGF1 and HGF did not affect DNMT1 mRNA levels (data no shown), so it is likely that its effect is on regulating the protein, including protein stability. It has been reported that AKT phosphorylates and inactivates GSK $3 \beta$, which recruits $\beta \operatorname{TrCP}$ to degrade substrate protein through a ubiquitin-proteasome pathway and also stabilize DNMT1 and maintains DNA methylation $(26,27)$. To explore this possibility, HepG2 cells were treated with either IGF1 or the GSK3 $\beta$ inhibitor LiCl. Similar to IGF1, $20 \mathrm{M} \mathrm{LiCl}$ upregulated DNMT1 expression and GSK3 $\beta$ phosphorylation in a manner independent of IGF1R $\beta$ activation (Fig. 4A). pGSK3 $\beta$ (Ser9), an inactive form, gradually increased with DNMT1 expression in HepG2 cells treated with SB-216763, a GSK $3 \beta$ inhibitor, for $4 \mathrm{~h}$ or longer (Fig. 4B). MG132, a proteasome-specific inhibitor, also increased DNMT1 protein levels in HepG2 cells (Fig. 4C). Fig. 4D shows that the synthesis inhibitor cycloheximide (CHX) decreased DNMT1 protein levels in a time-dependent manner; this decrease was rescued by IGF1 in HepG2 cells. These results indicate that IGF1-induced DNMT1 protein upregulation involves the GSK3 $\beta$-mediated protein degradation pathway.

Next, HA-ubiquitin or DNMT1 plasmids were transiently transfected into HepG2 cells and co-immunoprecipitation (co-IP) was performed with either anti-HA or anti-DNMT1 antibodies, respectively (Fig. 4E and F). As expected, results from western blotting showed that ubiquitin protein interacts with DNMT1, $\beta \operatorname{TrCP}$ and GSK3 $\beta$ (Fig. $4 \mathrm{E}$, lane 5), and this interaction was abrogated by treatment with IGF1 (Fig. 4E, lane 6). The reduced interaction between DNMT1 and the ubiquitin- $\beta$ TrCP-GSK3 $\beta$ complex induced by IGF1 treatment was confirmed by reverse co-IP with an anti-DNMT1 antibody (Fig. 4F, lanes 5 and 6). Immunofluorescence (IF) staining indicated that the active form of GSK3 $\beta$ co-localized with DNMT1 in the nucleus, which was abrogated by IGF1 treatment, resulting in DNMT1 upregulation (Fig. 4G). Consistent 
A

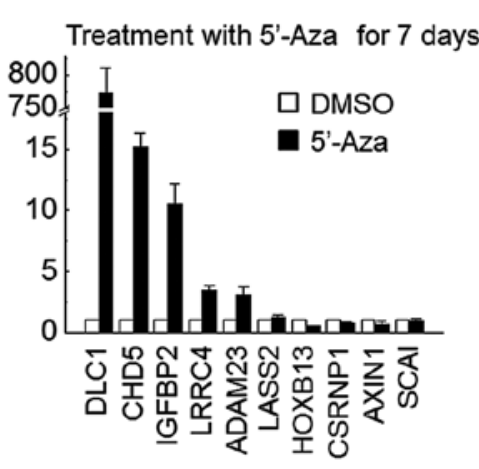

E

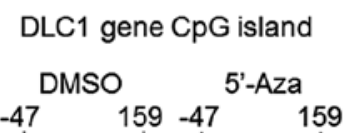

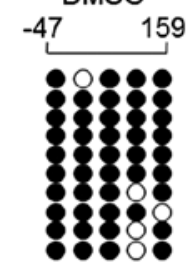

$45 / 50$
B

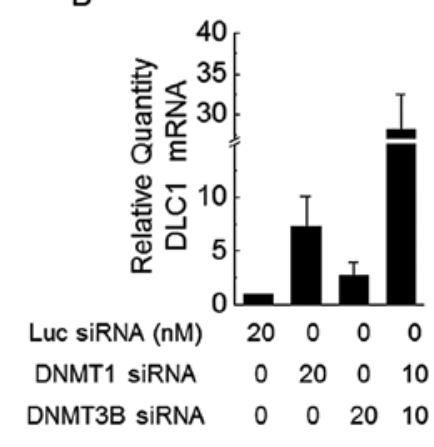

F

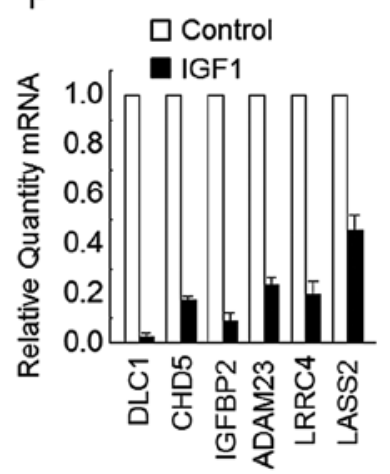

C

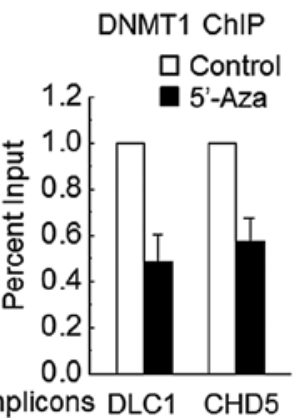

Amplicons DLC1 CHD5

G

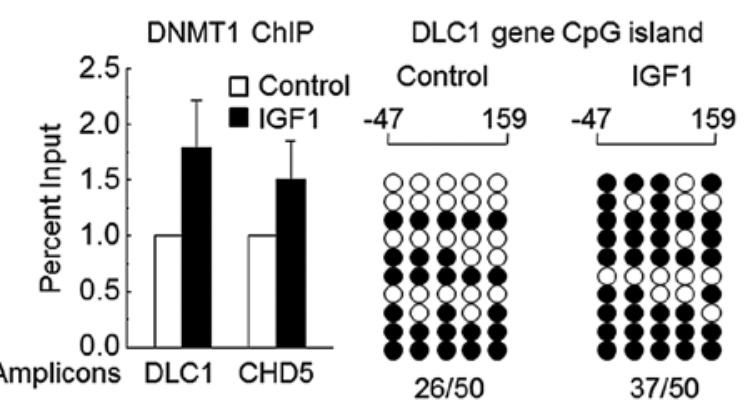

Figure 5. Analysis of the expression and methylation of the indicated DNMT target genes in HepG2 cells. (A) mRNA expression of several DNMT target genes was detected by real-time qPCR in HepG2 cells treated with 5'-Aza for 7 days. (B) DLC1 mRNA expression detected by real-time qPCR on the silencing of DNMT genes in HepG2 cells. (C and D) ChIP assays of (C) DNMT1 and (D) DNMT3B binding at the DLC1 and CHD5 promoters was decreased by 5'-Aza treatment for 7 days. (E) Ten segments selected for sequencing and alignment of $D L C 1$ in 5'-Aza treated HepG2 cells. White and black circles represent unmethylated and methylated CpGs, respectively. (F) mRNA expression of several DNMT target genes was detected by qPCR in HepG2 cells treated with IGF1 for 7 days. (G) Increases in DNMT1 binding to DLC1 and CHD5 promoters were determined by ChIP assays. (H) Ten segments selected for sequencing and alignment of $D L C 1$ in IGF1 treated HepG2 cells.

with this observation, Fig. $4 \mathrm{H}$ shows that siRNA-mediated IGF1R knockdown (KD) substantially blocked IGF1-induced pI3K-AKT pathway activation and DNMT1 expression. Finally, we evaluated the relationship between pAKT activation and DNMT1 expression in clinical HCC samples. Results from western blotting revealed that DNMT1 expression was markedly increased in 59.4\% (19/32) samples compared with adjacent normal tissue. pAKT was noticeably increased in 71.9\% (23/32) of HCC samples (Fig. 4I). Among the samples, 16 cases showed upregulation of both DNMT1 and pAKT. These results indicate that IGF-IGF1R $\beta$ activation upregulates DNMT1 expression via an AKT-GSK3 $\beta$-mediated ubiquitinproteasome pathway in HCC.

IGF1 suppresses TSGs transcription via DNA hypermethylation. Finally, we focused on several potential TSGs (DLCl, CHD5, IGFBP2, ADAM23, LRCC4, LASS2, SCAI, HOXB13, $A X I N 1$ and CSRNPI) commonly methylated in HCC, and performed real-time qPCR to detect their expression in HepG2 cells treated with 5'-Aza. For HepG2 cells exposed to 5'-Aza for 7 days, DLC1, CHD5, IGFBP2, ADAM23 and $L R C C 4$ were markedly upregulated (Fig. 5A). We also found that either DNMT1 or DNMT3B siRNA obviously upregulated $D L C 1$ expression, and siRNAs for both DNMT1 and DNMT3B together dramatically increased $D L C 1$ mRNA levels (Fig. 5B). ChIP assays showed that $D L C 1$ and CHD5 promoters were bound by DNMT1 and DNMT3B, and this binding was abrogated by 5'-Aza (Fig. 5C and D). Bisulfate sequencing assays showed that $\mathrm{CpG}$ loci of $D L C l$ are heavily methylated in wild-type $\mathrm{HepG} 2$ cells and the DNA methylation level was obviously reduced by 7 days of treatment with 5'-Aza (Fig. 5E). These results demonstrate that DNMT1 and DNMT3B cooperate in methylation of the $\mathrm{DLCl}$ and $\mathrm{CHD} 5$ promoters.

As expected, the mRNA expression of certain DNMTs target TSGs (DLC1, CHD5, IGFBP2, ADAM23, LRRC4 and LASS2) was significantly downregulated in HepG2 cells exposed to IGF1 for 7 days (Fig. 5F). Consistent with this observation, binding of DNMT1 to either $D L C 1$ or $C H D 5$ CpG loci was enhanced in HepG2 cells treated with IGF1 for 7 days (Fig. 5G). Fig. 5H shows that DNA methylation levels for the $D L C 1$ promoter were increased by 7 days of treatment with IGF1. These results clearly show that IGF1 suppresses TSG transcription, at least in part, through DNA methylation mechanisms.

\section{Discussion}

Emerging evidence suggests a relationship between DNA hypermethylation and hepatocarcinogenesis (28). However, the mechanistic and biological significance of DNA hypermethylation in human HCC is unclear. DNMTs promote liver cancer cell proliferation by regulating the expression and activity of cell cycle proteins. Moreover, siRNA interference 


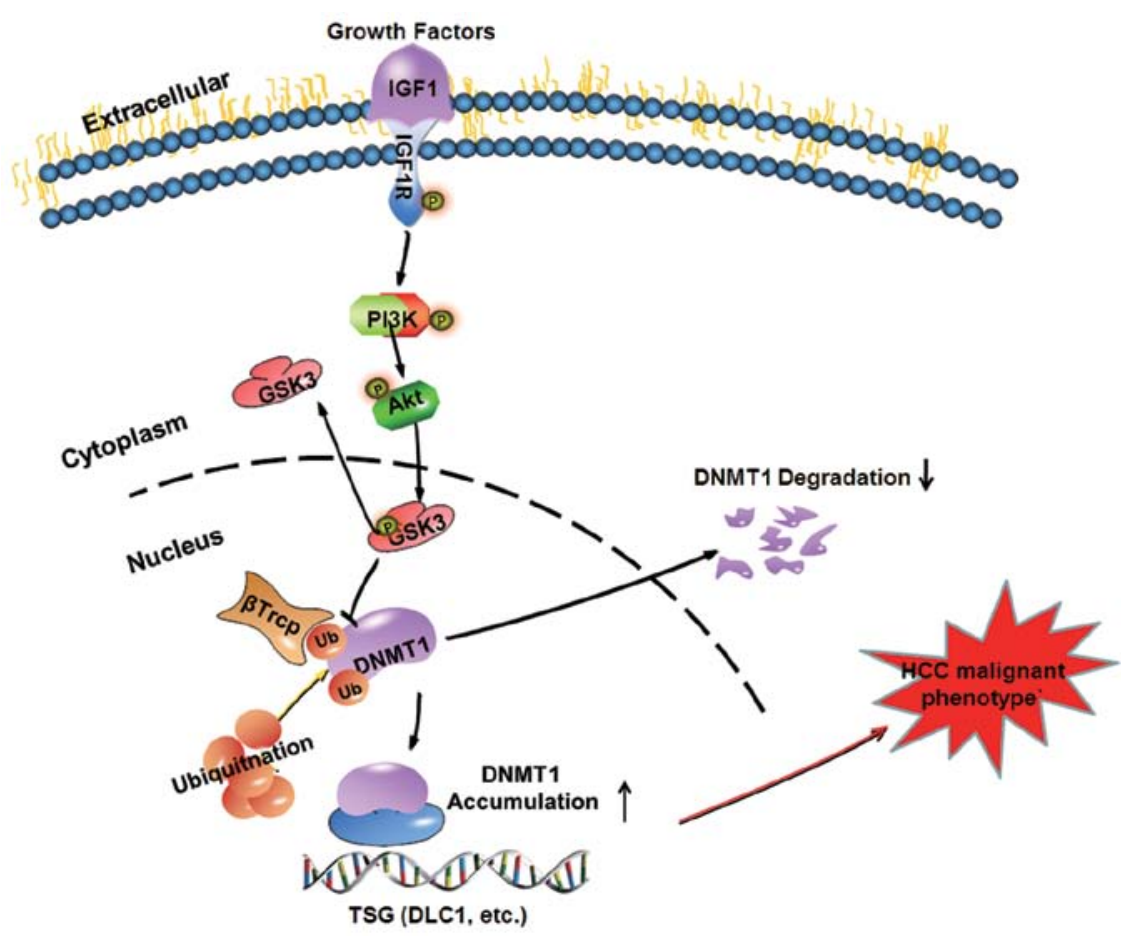

Figure 6. Proposed model to illustrate nuclear accumulation of DNMT1 by IGF1-induced AKT-GSK3 $\beta-\beta \operatorname{TrCP}$ signaling leading to TSG promoter hypermethylation and tumorigenesis. IGF1 induces DNMT1 protein accumulation in the nucleus through AKT-GSK3 $\beta-\beta \operatorname{TrCP}$ signaling. $\beta \operatorname{TrCP}$ is an E3 ubiquitin ligase that specifically interacts with and degrades DNMT1. IGF1 induces activation of AKT, then promotes GSK3 $\beta$ phosphorylation at Ser9 to form inactive GSK3 $\beta$, which subsequently attenuates the ability of $\beta$ TrCP to degrade DNMT1 protein. DNMT1 protein binds to the promoters of various TSGs and results in promoter hypermethylation and transcriptional silencing, which ultimately leads to tumorigenesis and poor prognosis.

of DNMT1 or/and DNMT3B significantly inhibits liver cancer cell proliferation, and 5'-Aza can inhibit HepG2 cell migration and the growth of established tumors in mice. Overall, DNA methylation is a potential target for effective treatment of HCC. The HCC control potential of chemotherapy is limited by the tolerance of hepatoma cells to drugs. Kurita et al (29) reported that double knockdown of DNMT1 and DNMT3B sensitizes HCC cells to TRAIL-mediated apoptosis, indicating the potential importance of aberrant DNA hypermethylation in the chemotherapy tolerance of HCC. This seems to be related to silencing of certain TSGs by DNA hypermethylation, which controls resistance to drugs in the liver. The function of 5'-Aza in eliminating methylation is explicit, but its clinical use as an anti-neoplastic agent is limited by its toxicity. However, demethylation could be a novel supplementary therapy that might enhance the curative effect of conventional chemotherapy in HCC; this will be an important issue for clinical investigation.

It is now thought that relapse and metastasis severely limit rehabilitation and HCC prognosis after traditional treatments such as surgery and radio/chemotherapy. It has been confirmed that tumor-derived growth factors are associated with recurrence following radical hepatectomy in HCC. Our results reveal a previously unknown epigenetic mechanism: as one component of the tumor microenvironment, growth factors can promote HCC malignant phenotype through DNMT1 upregulation, resulting in nuclear DNMT1 accumulation and hypermethylation of TSG promoters. The mechanism and significance of DNMT1 overexpression induced by growth factors remain poorly understood. We identified a novel growth factor pathway that involves de novo DNA meth- ylation and tumor suppressor silencing via upregulation of DNMT1 protein expression. The significance of IGF-IGF1R in hepatocarcinogenesis has been confirmed (30) and involves multiple stages and genes, as well as numerous signaling pathways, such as MAPK and PI3K/AKT (5). The present study provides direct evidence that activation of the IGF1-IGF1R $\beta$ axis induces DNMT1 protein accumulation in the nucleus, primarily through PI3K-AKT signaling. Co-IP assays revealed that DNMT1 interacts with $\beta$ TrCP and GSK $3 \beta$ in HepG 2 cells, which involves the ubiquitin-mediated protein degradation pathway. IGF1 protects DNMT1 stability through abrogation of the DNMT1- $\beta$ TrCP-GSK3 $\beta$ complex. These findings are in agreement with the notion that DNMT1 protein ubiquitination and proteasome-mediated degradation depend on a destruction domain mapped to the $\mathrm{N}$-terminal 120 amino acids that is also important for $\beta \operatorname{TrCP}$ interaction $(22,31)$. In clinical samples, high correlation between PI3K-AKT activation and DNMT1 upregulation was observed. This confirms the crucial role of pI3K-AKT in IGF1-induced DNMT1 upregulation. This is similar to the report that NNK activates AKT, inhibiting GSK $3 \beta-\beta \operatorname{TrCP}$ complex formation and thereby attenuating proteasomal degradation of DNMT1 in lung cancer cells (22). We investigated whether a similar mechanism applies to other growth factors and IGF1. The results show that IGF2, EGF and HGF activate AKT phosphorylation. It suggests that protein stability may be the common mechanism in growth factorinduced DNMT1 upregulation.

Although some studies have found increased expression of DNMTs in HCC, the mechanistic significance is unknown at present. Our studies answer this question by identifying a novel mechanism in which DNMT1 overexpression depends 
on extracellular cytokines, similar to the mechanism for NNK regulation of DNMT1 expression via the ubiquitin protein degradation pathway (22). These findings further confirm that epigenetic modifications such as DNA methylation play an indispensable role in regulating the expression of target genes and controlling cell proliferation, differentiation and functional specialization in hepatocarcinogenesis. We used a promoter array to identify genome-wide methylation profiles in $\mathrm{HCC}$ cells and found significant hypermethylation of $\mathrm{CpG}$ sites for a series of TSGs (DLC1, CHD5, IGFBP2, LRRC4 and $L A S S 2$ ) with notable biological significance in hepatocarcinogenesis. A putative tumor suppressor gene, $D L C 1$, was identified and mapped to chromosome 8p21.3-22, a region suspected to harbor tumor suppressor genes and recurrently deleted in HCC, prostate, lung and breast cancers. The present study provides evidence that DNMT1 and DNMT3B are directly co-located on $D L C 1 \mathrm{CpG}$ sites, resulting in promoter hypermethylation. Significant downregulation of DLC1 mRNA expression in HCC tissues was associated with enhanced $\mathrm{CpG}$ hypermethylation. DNA methylation is another crucial mechanism for DLC1 inactivation besides genetic regulation. Mapping to 1p36, chromodomain helicase DNA binding protein 5 ( $\mathrm{CHD5}$ ) is a tumor suppressor that serves as a master regulator of a tumor suppressive network controlling proliferation, apoptosis and senescence. The present study provides direct evidence that DNMT1 and DNMT3B bind to the CpG loci of $D L C 1$ and $C H D 5$, leading to promoter hypermethylation and transcriptional silencing. IGF1 significantly suppressed mRNA expression of certain TSGs, accompanied by increased accumulation of DNMT1 at DLC1 and CHD5 CpG loci in HepG2 cells. These results clearly show that IGF1 suppresses TSGs transcription via DNA methylation, at least in part.

In conclusion, our results point to a novel epigenetic mechanism for growth factor-mediated repression of TSG transcription that involves DNA methylation (Fig. 6). IGF1 induces activation of AKT, then promotes GSK $3 \beta$ phosphorylation at Ser9 to form inactive GSK3 $\beta$, which subsequently attenuates the ability of $\beta \mathrm{TrCP}$ to degrade DNMT1 protein. DNMT1 protein binds to the promoters of various TSGs and results in promoter hypermethylation and transcriptional silencing, which ultimately leads to tumorigenesis and poor prognosis. Our results not only enriched our understanding of a novel epigenetic mechanism of growth factor in the regulation of TSG transcription by DNA methylation but also provided a novel target for HCC diagnosis and prognosis.

\section{Acknowledgements}

The present study was supported by grants from the National Natural Science Foundation of China (no. 81172286 and no. 81372618), the '973'Program (no. 2013CB910803) and the National Key Sci-Tech Special Project of China (no. 2012ZX10002011-005). We appreciate the valuable comments from other members of our laboratories.

\section{References}

1. Jemal A, Bray F, Center MM, Ferlay J, Ward E and Forman D Global cancer statistics. CA Cancer J Clin 61: 69-90, 2011.
2. Hayat MJ, Howlader N, Reichman ME and Edwards BK: Cancer statistics, trends, and multiple primary cancer analyses from the Surveillance, Epidemiology, and End Results (SEER) Program. Oncologist 12: 20-37, 2007.

3. Brenner H, Stegmaier C and Ziegler H: Long-term survival of cancer patients in Germany achieved by the beginning of the third millenium. Ann Oncol 16: 981-986, 2005.

4. Kishi Y,Hasegawa K, Sugawara Y and Kokudo N: Hepatocellular carcinoma: current management and future developmentimproved outcomes with surgical resection. Int J Hepatol 2011: 728103, 2011.

5. Breuhahn K, Longerich T and Schirmacher P: Dysregulation of growth factor signaling in human hepatocellular carcinoma. Oncogene 25: 3787-3800, 2006.

6. Clendenen TV, Arslan AA, Lokshin AE, et al: Temporal reliability of cytokines and growth factors in EDTA plasma. BMC Res Notes 3: 302, 2010.

7. Zhao B, Zhang C, Forsten-Williams K, Zhang J and Fannon M: Endothelial cell capture of heparin-binding growth factors under flow. PLoS Comput Biol 6: e1000971, 2010.

8. Barbara L, Benzi G, Gaiani S, et al: Natural history of small untreated hepatocellular carcinoma in cirrhosis: a multivariate analysis of prognostic factors of tumor growth rate and patient survival. Hepatology 16: 132-137, 1992.

9. Chitnis MM, Yuen JS, Protheroe AS, Pollak M and Macaulay VM: The type 1 insulin-like growth factor receptor pathway. Clin Cancer Res 14: 6364-6370, 2008.

10. Whittaker S, Marais R and Zhu AX: The role of signaling pathways in the development and treatment of hepatocellular carcinoma. Oncogene 29: 4989-5005, 2010.

11. Kurmasheva RT and Houghton PJ: IGF-I mediated survival pathways in normal and malignant cells. Biochim Biophys Acta 1766: 1-22, 2006.

12. Shimizu M, Shirakami Y, Sakai H, et al: EGCG inhibits activation of the insulin-like growth factor (IGF)/IGF-1 receptor axis in human hepatocellular carcinoma cells. Cancer Lett 262: 10-18, 2008.

13. Pi-Chieh Wang K, Lee LM, Lin TJ, et al: Gene transfer of IGF1 attenuates hepatocellular apoptosis after bile duct ligation. J Surg Res 167: 237-244, 2011

14. Jones PA and Baylin SB: The fundamental role of epigenetic events in cancer. Nat Rev Genet 3: 415-428, 2002.

15. Ting AH, Jair KW, Suzuki H, Yen RW, Baylin SB and Schuebel KE: $\mathrm{CpG}$ island hypermethylation is maintained in human colorectal cancer cells after RNAi-mediated depletion of DNMT1. Nat Genet 36: 582-584, 2004.

16. Patra SK, Patra A, Zhao H and Dahiya R: DNA methyltransferase and demethylase in human prostate cancer. Mol Carcinog 33: 163-171, 2002.

17. Saito Y,Kanai Y, Nakagawa T, et al: Increased protein expression of DNA methyltransferase (DNMT) 1 is significantly correlated with the malignant potential and poor prognosis of human hepatocellular carcinomas. Int J Cancer 105: 527-532, 2003.

18. Girault I, Tozlu S, Lidereau R and Bieche I: Expression analysis of DNA methyltransferases 1,3A, and 3B in sporadic breast carcinomas. Clin Cancer Res 9: 4415-4422, 2003.

19. Etoh T, Kanai Y, Ushijima S, et al: Increased DNA methyltransferase 1 (DNMT1) protein expression correlates significantly with poorer tumor differentiation and frequent DNA hypermethylation of multiple $\mathrm{CpG}$ islands in gastric cancers. Am J Pathol 164: 689-699, 2004.

20. Lin RK, Hsu HS, Chang JW, Chen CY, Chen JT and Wang YC: Alteration of DNA methyltransferases contributes to $5^{\prime} \mathrm{CpG}$ methylation and poor prognosis in lung cancer. Lung Cancer 55: 205-213, 2007.

21. Lambert MP, Paliwal A, Vaissiere T, et al: Aberrant DNA methylation distinguishes hepatocellular carcinoma associated with HBV and HCV infection and alcohol intake. J Hepatol 54: 705-715, 2011.

22. Lin RK, Hsieh YS, Lin P, et al: The tobacco-specific carcinogen NNK induces DNA methyltransferase 1 accumulation and tumor suppressor gene hypermethylation in mice and lung cancer patients. J Clin Invest 120: 521-532, 2010.

23. Dong C, Yuan T, Wu Y, et al: Loss of FBP1 by Snail-mediated repression provides metabolic advantages in basal-like breast cancer. Cancer Cell 23: 316-331, 2013. 
24. Tiwari N, Tiwari VK, Waldmeier L, et al: Sox4 is a master regulator of epithelial-mesenchymal transition by controlling Ezh2 expression and epigenetic reprogramming. Cancer Cell 23: 768-783, 2013.

25. Au SL, Wong CC, Lee JM, Wong CM and Ng IO: EZH2-mediated $\mathrm{H} 3 \mathrm{~K} 27 \mathrm{me} 3$ is involved in epigenetic repression of deleted in liver cancer 1 in human cancers. PLoS One 8: e68226, 2013.

26. Taketo MM: Shutting down Wnt signal-activated cancer. Nat Genet 36: 320-322, 2004.

27. Sun L, Zhao H, Xu Z, et al: Phosphatidylinositol 3-kinase/protein kinase B pathway stabilizes DNA methyltransferase I protein and maintains DNA methylation. Cell Signal 19: 2255-2263, 2007.
28. Ma X, Wang YW, Zhang MQ and Gazdar AF: DNA methylation data analysis and its application to cancer research. Epigenomics 5: 301-316, 2013.

29. Kurita S, Higuchi H, Saito Y, et al: DNMT1 and DNMT3b silencing sensitizes human hepatoma cells to TRAIL-mediated apoptosis via up-regulation of TRAIL-R2/DR5 and caspase-8. Cancer Sci 101: 1431-1439, 2010.

30. Liu X, Jiang W, Aucejo F, et al: Insulin-like growth factor I receptor beta expression in hepatocellular carcinoma. Hum Pathol 42: 882-891, 2011.

31. Agoston AT, Argani P, Yegnasubramanian S, et al: Increased protein stability causes DNA methyltransferase 1 dysregulation in breast cancer. J Biol Chem 280: 18302-18310, 2005. 